\title{
NUMERICAL ANALYSIS OF OSCILLATING FLOW AROUND A CYLINDER
}

\author{
Renata Gnatowska \\ Institute of Thermal Machinery, Czestochowa University of Technology \\ Częstochowa, Poland \\ gnatowska@imc.pcz.czest.pl
}

\begin{abstract}
This paper presents the numerical model of the measuring stand - the wind tunnel, in which there is fixed a cylinder with a turbulent inlet oscillating stream. The aim of this work is the juxtaposition and comparison of characteristic values concerning the oscillating turbulent flow around the cylinder, obtained from the experiment conducted in the wind tunnel at the Institute of Thermal Machinery with the data obtained as a result of numerical modelling of unsteady phenomena. The model discussed in this paper was created using a commercial program ANSYS FLUENT that is used for mathematical modelling of flow and heat transfer processes. The expected outcome of this study is possibility of the numerical modelling of the stand concerning the analogous unsteady flows without significant investment of time. Comparison of longitudinal and transverse velocity profiles in aerodynamic wake and the pressure coefficient distributions on the cylinder surface show similarities between experimental and numerical studies.
\end{abstract}

Keywords: mathematical modelling, unsteady flow phenomena, circular cylinder

\section{Introduction}

Cylindrical structures exposed to the flow are basically present in all areas of engineering and in the environment. The flow is associated with unsteady vortex shedding and this special feature has a dominant influence on the flow behaviour itself, on the loading of cylindrical structures, which is often unsteady [1]. In the present paper attention is focused on the long rigid stationary circular cylinder exposed to an oscillatory incident flow. This configuration has many applications in industrial processes. The topic is of direct relevance with regard to offshore platforms, pipelines immersed in waves, heat exchangers, power cables and civil engineering structures in oscillating wind flow [2]. Studies relevant to industrial failures and solutions proposed for design improvement confirm the aerodynamic origin of the problem and are linked to fundamental work in fluid dynamics. A wide variety of configurations is possible ranging from infinitely long cylinders in uniform approach flow to the cylinder in shear inflow like boundary layers, cylinders oblique to the approach flow, various cross-sectional geometry, cylinders having short aspect ratios, etc. 
The Reynolds hypothesis for turbulent flow assumes that the velocity $(u, v, w)$ and pressure $p$ are decomposed into a mean component and a fluctuating component [1]. The fluctuating flow field component is a direct result of an alternate vortex generation and shedding into the body wake. On the other hand, the organized time-dependent fluctuations of the flow surrounding the bluff body can be introduced through the periodical external disturbances. The works concerned with vortex shedding from cylinders immersed in an oscillatory incident flow have been reviewed by Griffin and Hall [3], Armstrong et al. [4] and analyzed also in the Institute of Thermal Machinery by Jarża and Podolski $[5,6]$. It occurs when the incident velocity field $U_{0}(t)$ has a periodical component characterized by a properly matched frequency $f_{0}$ and amplitude $A$.

The model discussed in this paper was created using a commercial program ANSYS FLUENT that is used for mathematical modelling of flow and heat transfer processes. The expected outcome of this study is that it is possible in a way that doesn't require significant investment of time, numerical modelling of analogous stand concerning issues of unsteady flows. The present numerical research has been undertaken in order to explain the turbulent structure of the two-dimensional wake-flow may be affected by the incident flow oscillations. Special attention is focused on the mathematical equivalent of a wind tunnel located at the Institute of Thermal Machinery of Czestochowa University of Technology. The unsteady flow field is analysed on the basis of a complete set of mean velocity profiles in the near-wake region. A matter of interest of the present study has also been related to mean surface pressure components evolution around a rigid circular cylinder in oscillatory inflow. The results obtained from unsteady Reynolds-averaged Navier-Stokes (URANS) are compared with experimental data available in the Institute of Thermal Machinery performed by Jarża and Podolski [5].

\section{Computational model}

This paper is dedicated to the issue of the numerical model of a measuring station - wind tunnel, in which a cylinder is mounted with oscillating turbulent inflow. Verification calculations were used for experiment carried out in an open-circuit wind tunnel at the Institute of Thermal Machinery of Czestochowa University of Technology. The model discussed in this work was created based on the computational grid generated in GAMBIT, which cooperates with ANSYS FLUENT commercial code.

The first step was to create a model of the measuring stand. Scheme of this geometry, the measuring stand is shown in Figure 1. The test section was $0.4 \times 0.4 \mathrm{~m}$ $(W \times H)$ and $\approx 4 \mathrm{~m}$ long. The circular cylinder of $0.078 \mathrm{~m}$ in diameter and $0.4 \mathrm{~m}$ long was located $1.2 \mathrm{~m}$ from the entrance to the working section, perpendicular to the free-stream direction. Proper setting up the boundary conditions at the inlet and outlet of the model allows for the achievement of an effect of real conditions. The following boundary conditions are used in calculations: at the inlet VELOCITY 
INLET, at the outlet PRESSURE OUTLET, for the upper and lower walls and the cylinder surface - WALL condition. The blockage ratio, defined as the ratio of the height of the body to the height of computational domain, is about 0.19 . For computation purposes, the flow domain is divided into a number of square cells. The grid is clustered near the object and the spacing is increased to a proper ratio of 1.2 away from the object surface. The first cell adjacent to the walls has been set with respect to the criteria required for the individual near-wall treatment. The grid independent solution has been obtained for 106000 cells by systematically refining the entire mesh in each direction, increasing the number of nodes by about $50 \%$ [7].

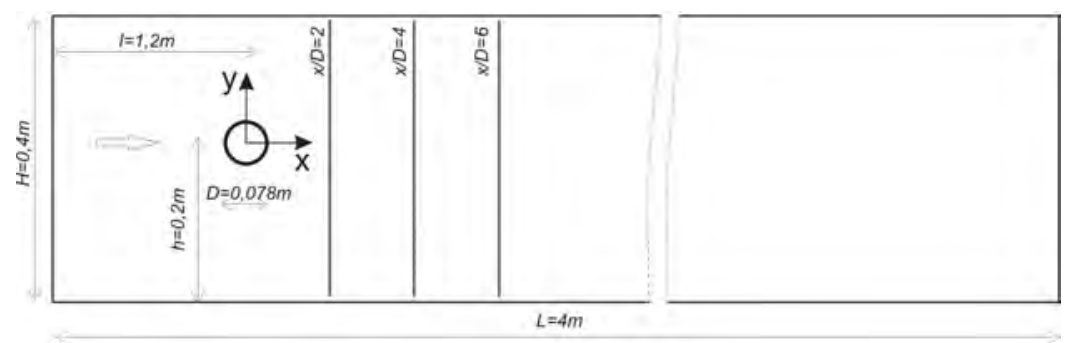

Fig. 1. Schematic sketch of the flow considered [5]

The paper assumes the two-dimensional character of the phenomenon, even though the aerodynamic wake at a certain distance from the cylinder is a longitudinal component of vorticity, making three-dimensional flow. The validity of the assumption will be verified with experimental data. But computational effort to three-dimensional analysis made it impossible to take such tests in the context of the present work. In considerations a decision was made to use the structural grid.

The unsteady nature of the considered flow requires application of the calculation procedure, which allows one to follow changes in the flow as a function of time. For the calculation of the present study a decision was made to use the k- $\varepsilon$ turbulence model. Writing the equations in the form of averaged phase [8-10] in combination with the turbulence model k- $\varepsilon$ RNG version of the method used allows one to qualify a group URANS (unsteady Reynolds-averaged Navier-Stokes). The kinetic energy $k$ and the dissipation kinetic energy $\varepsilon$ are calculated by the equations of transport in which are the phase-averaged parameters $\diamond$ :

$$
\begin{gathered}
\frac{\partial\langle\boldsymbol{k}\rangle}{\partial \boldsymbol{t}}+\frac{\partial\left[\left\langle\boldsymbol{U}_{i}\right\rangle\langle\boldsymbol{k}\rangle\right]}{\partial \boldsymbol{x}_{i}}=\frac{\partial}{\partial \boldsymbol{x}_{i}}\left[\frac{\left\langle v_{T}\right\rangle}{\sigma_{\boldsymbol{k}}} \frac{\langle\boldsymbol{k}\rangle}{\partial \boldsymbol{x}_{i}}\right]+\boldsymbol{P}_{\boldsymbol{k}}-\langle\varepsilon\rangle \\
\frac{\partial\langle\boldsymbol{\varepsilon}\rangle}{\partial \boldsymbol{t}}+\frac{\partial\left[\left\langle\boldsymbol{U}_{\boldsymbol{i}}\right\rangle\langle\boldsymbol{\varepsilon}\rangle\right]}{\partial \boldsymbol{x}_{\boldsymbol{i}}}=\frac{\partial}{\partial \boldsymbol{x}_{i}}\left[\frac{\left\langle\boldsymbol{v}_{T}\right\rangle}{\sigma_{\varepsilon}} \frac{\langle\boldsymbol{\varepsilon}\rangle}{\partial \boldsymbol{x}_{i}}\right]+\boldsymbol{C}_{\varepsilon_{1}} \boldsymbol{P}_{\boldsymbol{k}} \frac{\langle\boldsymbol{\varepsilon}\rangle}{\langle\boldsymbol{k}\rangle}-\boldsymbol{C}_{\varepsilon_{2}} \frac{\langle\varepsilon\rangle^{2}}{\langle\boldsymbol{k}\rangle}+\boldsymbol{R}
\end{gathered}
$$

where $\boldsymbol{P}_{\boldsymbol{k}}$ - production of turbulence kinetic energy, expressed as: 


$$
\boldsymbol{P}_{k}=C_{\mu}\langle\varepsilon\rangle S^{2}
$$

where $\boldsymbol{S}$ - deformation tensor is defined as:

$$
\boldsymbol{S}=\frac{\langle\boldsymbol{k}\rangle}{\langle\boldsymbol{\varepsilon}\rangle} \sqrt{\frac{\mathbf{1}}{\mathbf{2}}\left[\frac{\partial\left\langle\boldsymbol{U}_{i}\right\rangle}{\partial \boldsymbol{x}_{j}}+\frac{\partial\left\langle\boldsymbol{U}_{j}\right\rangle}{\partial \boldsymbol{x}_{i}}\right]^{2}} .
$$

The turbulent viscosity coefficient is expressed as:

$$
\left\langle v_{T}\right\rangle=v\left[1+\left(\frac{C_{\mu}}{v}\right)^{1 / 2} \frac{\langle k\rangle}{\langle\varepsilon\rangle^{1 / 2}}\right]^{2} .
$$

The value of $\boldsymbol{R}$ in the transport equation of turbulent dissipation of kinetic energy:

$$
R=\frac{C_{\mu} \cdot \rho \eta^{3}\left(1-\eta / \eta_{0}\right)}{1+\beta \eta^{3}} \cdot \frac{\langle\varepsilon\rangle^{2}}{\langle k\rangle}
$$

where: $\eta=\boldsymbol{S} \cdot\langle\boldsymbol{k}\rangle /\langle\varepsilon\rangle, \eta_{\mathbf{0}}=\mathbf{4 . 3 8}, \boldsymbol{\beta}=\mathbf{0 . 0 1 2}$ allows a reduction in the excessive growth of turbulent kinetic energy in the region of large gradient of mean velocity. For $\eta>\eta_{0}, \boldsymbol{R}$ assumes a negative value, which also increases the dissipation of turbulent kinetic energy, thus reducing the production of turbulent kinetic energy. Constants occurring in the equations are: $C_{1 \varepsilon}=1.42, C_{2 \varepsilon}=1.68, C_{\mu}=\mathbf{0 . 0 8 4 5}$, and Prandtl numbers respectively $\sigma_{\boldsymbol{k}}=\mathbf{0 . 7 1 7 8}, \sigma_{\varepsilon}=\mathbf{0 . 7 1 7 8}$ [7].

In order to solve the presented equations, one should define and implement boundary conditions. The oncoming stream (inlet condition of velocity) was thus approximately given by:

$$
U=\overline{U_{0}}\left(1+A \sin 2 \pi f_{0} t\right)
$$

where $U_{0}=5.5 \mathrm{~m} / \mathrm{s}, A=0.2 \cdot \mathrm{U}_{0}$ and $f_{0}=0 \sim 26 \mathrm{~Hz}$ is the driving frequency.

\section{Result and discussion}

The present research concentrates on the structure of the near wake, which together with the flow properties in the immediate vicinity of the cylinder wall combines random characteristics with a periodical form of organization. In the frame of numerical research, the turbulent structure of the two-dimensional wake-flow without and with incident flow oscillations was analysed. The measure and calculation were realized around the cylinder surface for a range of incident flow oscilla- 
tions, and presented only as 0 and $26 \mathrm{~Hz}$. The data obtained from the simulation: the average longitudinal (Figs. 2, 4) and transverse velocity profiles (Figs. 3, 5) in the wake behind the cylinder in selected sections: $x / D=2, x / D=4$ and $x / D=6$ were summarized with experimental data. The velocity profiles show the evolution of the aerodynamic wake. In the Figures $3 \mathrm{a}$ and $5 \mathrm{a}$ showing the transverse velocity for $x / D=2$ two opposite extremes can be seen, which is a clear effect of turbulence behind the body. In the area of close wake transverse velocity component marks its presence, which is the result of the circulation of the accompanying vortex structures, generated for the object. Alternating vortices have opposite directions of velocity, with values close to zero in the flow axis, while the extremes oscillate between 0.15 and -0.15 . When distance from the cylinder grows, the changes of longitudinal and transverse velocity are milder. In the following figures discrepancy is observed between the data obtained through simulation and experiment.

The relative depth of the wake for the inflow with oscillations is characterized by a velocity profile that is flatter than in the uniform inflow. The obtained values are divergent in the vicinity of the border of the aerodynamic wake, in areas there is a maximum of velocity.

a)

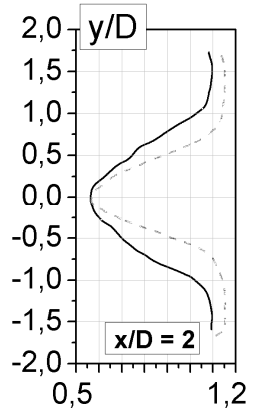

b)

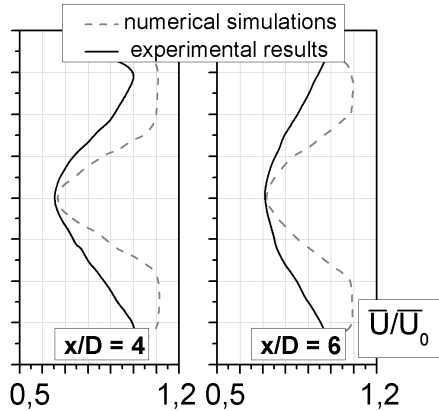

Fig. 2. Statement of longitudinal velocity profiles for different distances behind the circular cylinder: a) $x / D=2$, b) $x / D=4$, c) $x / D=6$ without incident oscillations $f_{0}=0 \mathrm{~Hz}$

a)

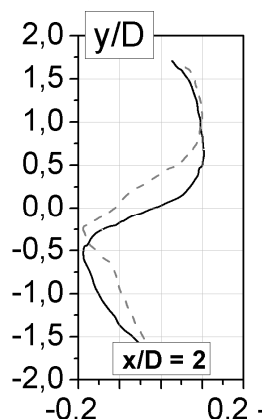

b)

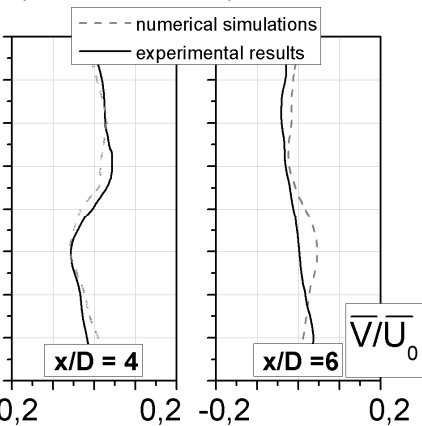

Fig. 3. Statement of transverse velocity profiles for different distances behind the circular cylinder: a) $x / D=2$, b) $x / D=4$, c) $x / D=6$ without incident oscillations $f_{0}=0 \mathrm{~Hz}$ 
a)

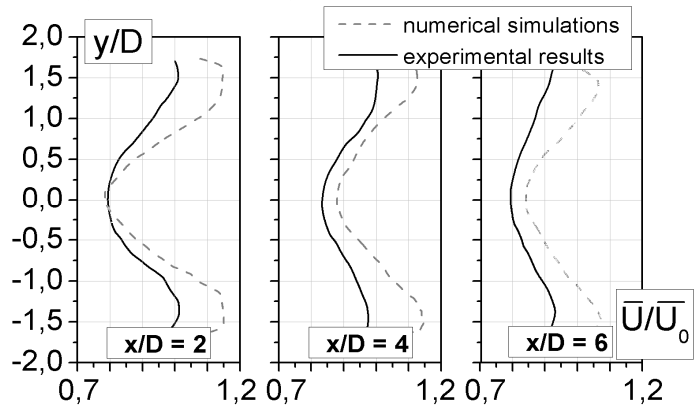

Fig. 4. Statement of longitudinal velocity profiles for different distances behind the circular cylinder: a) $x / D=2$, b) $x / D=4$, c) $x / D=6$ with incident flow oscillations $f_{0}=26 \mathrm{~Hz}$

a)

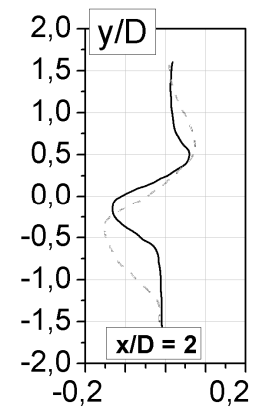

b)

c)

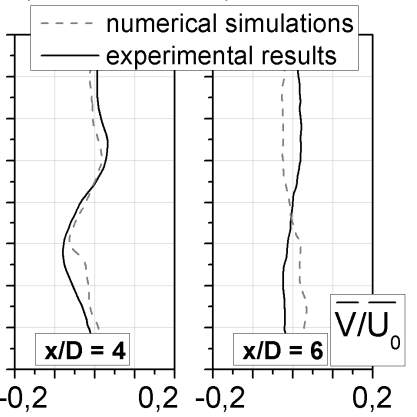

Fig. 5. Statement of transverse velocity profiles for different distances behind the circular cylinder: a) $x / D=2$, b) $x / D=4$, c) $x / D=6$ with incident flow oscillations $f_{0}=26 \mathrm{~Hz}$

The graph in Figure 6 shows the pressure coefficient distribution on the surface of the cylinder obtained on the basis of the experiment and as a result of numerical simulation.

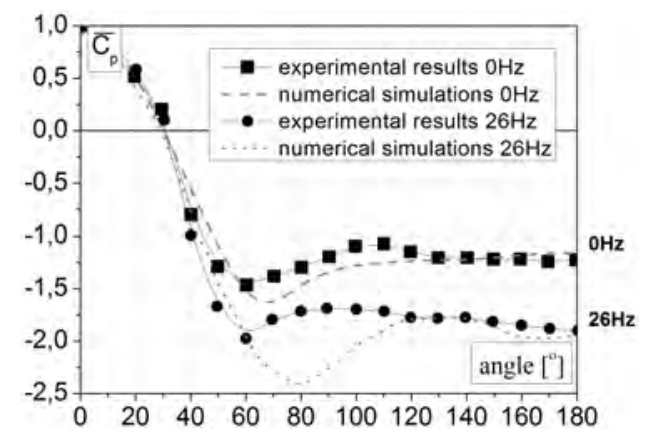

Fig. 6. Distributions of the surface pressure coefficient

The surface pressure coefficient distribution as a function of the position is defined as: 


$$
\overline{C_{p}}=\frac{\left(p_{s}-p_{s \infty}\right)}{\frac{1}{2} \rho{\overline{U_{\infty}}}^{2}}
$$

where: $\boldsymbol{p}_{\boldsymbol{s}}$ - mean static pressure on the cylinder surface; $\boldsymbol{p}_{\boldsymbol{s} \infty}$ - mean static pressure of the incident flow; $\rho$-density; $\overline{\boldsymbol{U}_{\infty}}$ - mean velocity of the incident flow.

In the front part of the cylinder the normalized pressure is almost independent of the inflow conditions. Important changes, however, are found in the rear as it was to be expected. The influence of the external flow oscillations appears to be limited to reducing the pressure coefficient at angular positions larger than $60^{\circ}$. In particular the pressure distributions on the back of the object reveal the maximum changes. Here good agreement is observed and performed a numerical simulation with conducted experiment. Difference in the region of the maximum pressure may result from the calculation of the flow, by the turbulence model, and thus slightly increased velocity in this section. This consequently provides the local pressure drop.

An important part of the study was performed the spectral analyses the influence the frequency oscillation on the formation of the vortex structures. It confirmed the correct lead numerical calculations. The frequency oscillation selected based on the experiment reflects a real impact on the frequency vortex shedding structures. It is characterized by the presence of the peak of vortices characterized by a higher proportion of energy (amplitude) than the peak describing the inlet oscillation. In addition, as shown in Figure 7, the frequency ratio of the inlet to the natural oscillation frequency vortex shedding structures is equal to 2 . This information proves that the modeled system at that frequency of inlet oscillation is equal to $26 \mathrm{~Hz}$, is in resonance frequency lock-on.

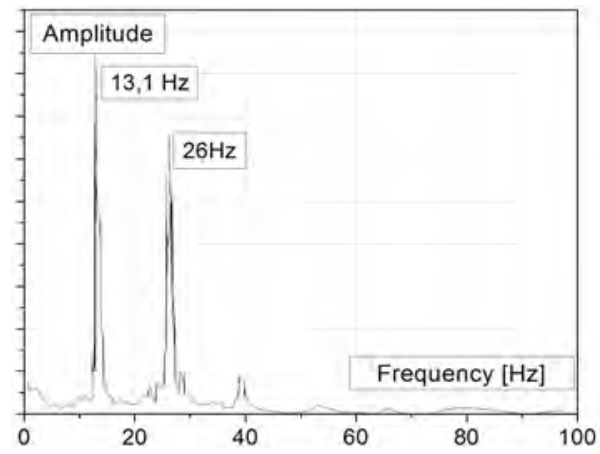

Fig. 7. Spectral analyses of frequency

\section{Conclusions}

The analysis of the obtained results makes it possible that the presented turbulence model can reflect the character of flow phenomena. It will also be noted that the two-dimensional nature of the flow was assumed, even though the aerodynamic 
wake at a certain distance from the cylinder there is a longitudinal component of vorticity, making a three-dimensional flow.

Overestimated or underestimated values of the parameters under consideration may also derive from the method of solving differential equations describing fluid motion, as well as probable changes in local turbulence intensity. Comparison of longitudinal and transverse velocity profiles in aerodynamic wake and pressure coefficient distributions on the cylinder surface show the similarity of flows, but should not be regarded as the results fully consistent with the experiment. In conclusion: 1. Properly modeled mesh for discussed flow. The density applied to the computational grid ensures a stable results of calculations; 2. Chosen turbulence model in the general assessment did not lead to full convergence of the results of calculations with experiment. Only to some extent observed similarity with the data obtained experimentally. But it is not an error caused by a poorly designed stand and a highly unsteady nature of the flow.

Furthermore, the correct execution of the experiment in a wind tunnel on the measurements of the characteristic parameters of objects here immersed in the turbulent flow. A more systematic investigation is needed to fully validate the methodology and numerical model for this turbulent flow.

\section{References}

[1] Elsner J.W., Drobniak S., Metrologia turbulencji przepływów, Seria Maszyny Przepływowe, tom 18, Ossolineum, 1995.

[2] Zdravkovich M.M., Flow around Circular Cylinders, Vol. 1, Fundamentals, First edition, Oxford University Press, New York 1997.

[3] Griffin O.M., Hall M.S., Review - vortex shedding lock-on and flow control in bluff body wakes, ASME Journal of Fluids Engng. 1991, 113, 526-537.

[4] Armstrong B.J., Barnes F.H., Grand J., The effect of perturbations on the flow over a cylinder, Phys. Fluids 1996, 29, 2095-2102.

[5] Jarza A., Podolski M., Turbulence structure in the vortex formation region behind a circular cylinder in lock-on conditions, European Journal of Mechanics B/Fluids 2004, 23, 535-550.

[6] Podolski M., Unsteady heat transfer from a circular cylinder in turbulent oscillation flow, Chemical and Process Eng. 2006, 27, 3/1, 695-705.

[7] ANSYS FLUENT 13.1 Theory Guide.

[8] Catalano P., Wang M., Iaccarino G., Moin P., Numerical simulation of the flow around a circular cylinder at high Reynolds numbers, Internat. J. of Heat and Fluid Flow 2003, 24, 463-469.

[9] Gnatowska R., Aerodynamic characteristics of two-dimensional sharp-edged objects in tandem arrangement, Arch. of Mech. 2008, 60, 6, 475-490.

[10] Bosch G., Rodi W., Simulation of vortex shedding past a square cylinder with different turbulence models, International Journal of Numerical Methods of Fluids 1998, 28, 601-616. 